\title{
Comparative Dose-Response Study of Stretching On Strength of Proximal (Hamstring) and Distal (Calf) Muscle
}

\author{
Khan', M., Quddus², N., Chawla ${ }^{3}$ C, Anwer ${ }^{4}$, S, \\ ${ }^{1}$ Physiotherapist, Ability Physiotherapy Clinic, New Delhi, India. \\ e-mail: masoodkhanphysio@gmail.com \\ ${ }^{2}$ Assistant Professor, Jamia Hamdard, New Delhi, India, email: nishat_quddus@yahoo.com \\ ${ }^{3}$ Director, Ability Physiotherapy Clinic, New Delhi, India, e-mail: chawlachandan@ hotmail.com \\ ${ }^{4}$ Asst. Professor, Padmashree Dr. D. Y. Patil College of Physiotherapy, Pimpri, Pune - 411018, \\ Maharashtra, India. e-mail: anwer_shahnawazphysio@rediffmail.com
}

\section{Abstract}

Objective: To compare the stretching induced strength changes between the proximal and distal groups of muscles. Methods: Twenty-eight recreationally active male individuals participated in this study. They were assigned into Group A for proximal muscle (hamstring) and group B for distal muscle (calf) with 14 subjects in each group. This study is an experimental trial of selfstretching for 2 minutes $\left(\mathrm{SS}_{2}\right)$. This stretch duration involved a 30 second stretch and a 20 second relaxation period, intermittently. Maximum Isometric Voluntary Contraction Force (MIVCF) was measured in both groups before (pretest) and immediately after (posttest) stretching. Change in pre-stretching and immediately post-stretching MIVCF was compared between the two groups. Results: Maximum Isometric Voluntary Contraction Force increased with $\mathrm{SS}_{2}$ in the hamstring by $1.31 \%$ and in calf muscle, it increased by $2.92 \%$. However, these changes were statistically insignificant between the two groups $(\mathrm{p}>0.05)$. Conclusion: Shorter stretching (2 minute) increases maximum isometric voluntary contraction force in both muscles but relatively more in the calf.

Key Words: Stretching induced, strength, MIVCF, strain gauge

\section{Introduction}

Stretching is traditionally used as part of a warm-up to increase flexibility or pain-free range of motion (ROM) about a joint in an attempt to promote better performances and/or reduce the risk of injury (Shellock and Prentice, 1985; Smith, 1994; Fowles et al., 2000). Athletic trainers and other rehabilitation professionals also recommend that their athletes or patients stretch before performing strengthening exercises or strength assessment tests (Bixler and Jonese, 1992). However, authors of recent systematic reviews and many original studies have suggested that pre exercise stretching may temporarily compromise a muscle's ability to produce force (Behm, 2001; Young and Elliott, 2001; Thacker et al., 2004; Shrier, 2004). It is possible that this short-term effect of stretching on muscle force production may affect the performance of various rehabilitation strengthening exercises. More importantly, pre exercise stretching may adversely affect the results obtained by muscle strength assessments and, in turn, influence a clinician's decisions regarding rehabilitation progression or return to play (McHugh and Nesse, 2007; Herda et al., 2009). Fowles et al (2000) reported that 30 minute of passive stretching reduces 
isometric torque by $28 \%$ and a $9 \%$ force deficit was still present at 1 hour post stretching. The reason for this reduction in muscle strength was thought to be mainly due to changes (decrease) in musculotendinous stiffness and altered motor control strategies (decreases in muscle activation) (Behm, 2001; Knudson et al., 2001).

In a study by Ryan et al (2008) it was suggested that shorter durations of stretching may not diminish muscle activation in the plantar flexors. In contrast, larger muscle groups such as the leg extensors have demonstrated reductions in muscle activation after 8 minute of stretching. Therefore, it is possible that the stretching induced decreases in muscle activation may be muscle specific, such that the suboptimal activation of the larger, proximal muscles may be amplified after stretching, whereas the near fully activated, distal muscles require longer durations of stretch to diminish muscle activation. Since then, no study has been done to test this hypothesis.

The present study involved the comparison of a proximal muscle and distal muscles regarding strength loss after stretching. It is hypothesized that selfstretching would result in transient acute reduction in muscle strength and stretch induced strength loss would be greater for proximal muscle (Hamstring muscle) as compared to distal muscle (Calf muscles).

\section{Materials \& Methods}

Subjects: A total of 28 male subjects with mean age 24.89 years were studied. Fourteen in group A participated in stretching and strength measurement of the proximal muscle (hamstring) and 14 in group B participated in stretching and strength measurement of distal muscle (calf). Inclusion criteria were; 20-30 years age group, subjects lacking at least 20 degrees of active knee extension with the hip in 90 degrees of flexion during active knee extension test for group $\mathrm{A}$ and for group B subjects having not more than 20 degrees of active dorsi-flexion in long sitting position with back supported against wall (Worrell, 1994). All the subjects were recreationally active (engaging in 1 to 5 hours of regular physical activity, not involving stretching exercises, per week). No one reported any current or ongoing neuromuscular diseases or musculoskeletal injuries specific to ankle, knee, or hip joints. None of the participants were competitive athletes. The study has the approval of Institutional Ethical Committee, Jamia Hamdard, New Delhi and written consent was obtained from all the participants. All subjects were recruited from Majeedia Hospital, New Delhi.

Study design: This was a pretest posttest experimental group designed to evaluate the effect of stretching on reduction of muscle strength and the comparison of a proximal muscle and distal muscles regarding strength loss after stretching. This study includes pre intervention assessment, Intervention and post intervention assessment.

Pre intervention assessment: Active range of motion and MIVCF were measured. All the procedures were explained to the subjects prior to assessment.

(A). AROM measurement: Active range of motion (AROM) was measured using Universal Goniometer. 
1. For Hamstring muscle: Active knee extension test was performed (Sullivan et al., 1992; Worrell et al., 1992). Subjects were made to lie supine. Hip joint was kept at 90 degrees of flexion. Immovable arm was placed parallel to long axis of the thigh. Fulcrum was placed at the lateral condyle of the femur. Movable arm was placed parallel to long axis of the leg. Subjects were asked to actively extend the leg up to maximum range. Lack in full extension at knee was noted.

2. For calf muscle: Subjects were made to lie in the supine position. Fulcrum of the goniometer was placed over the lateral malleolus. Immovable arm was placed parallel to the fibula. Movable arm was placed parallel to the lateral border of the foot. Subjects were asked to actively dorsiflex the foot from neutral ankle position (i.e. 90 degrees angle between foot and leg). The range, through which foot moved, was noted.

(B). Isometric strength measurement: The MIVCF was measured using electronic strain gauge device (Gold tech-model GTH, ENF Act 1985). It is a reliable and valid tool to measure muscle strength (Kennedy, 1965; Tiainen et al., 2004; Vivodtzev et al., 2006).

1. For Hamstring muscle: Subjects were made to sit on quadriceps table with arms resting on thighs. Thighs were tied to quadriceps table with the help of a strap. Hip joint was kept at 90 degrees of flexion and knee joint at 60 degrees of flexion. Strain gauge was attached to subject's leg. Then he was asked to flex the knee with maximum effort and hold it for 5 seconds (Fig 1).

2. For Calf muscle: Subjects were made to lie supine on a metallic couch. One roll of bed sheet was kept below the knee to put it in 20 degrees of flexion. Strain gauge was attached to subject's foot. Ankle was kept at neutral position, having 90 degrees angle between foot and leg.

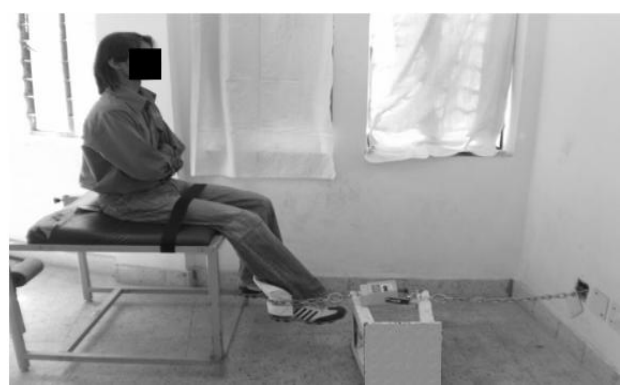

Figure 1: Measurement of isometric contraction of Hamstring muscle

Subjects were asked to plantar flex the foot with maximum effort and hold it for 5 seconds (Fig 2).

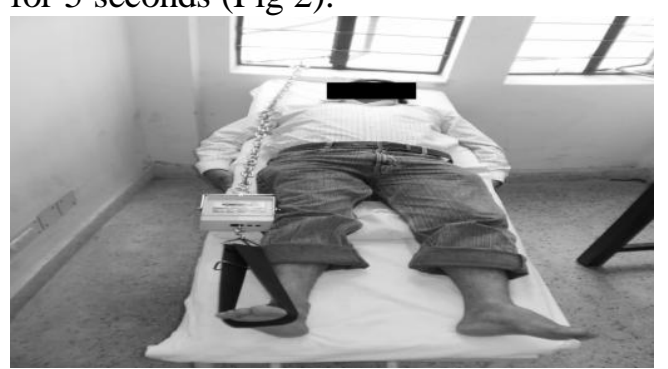

Figure 2: Measurement of isometric contraction of Calf muscle

Figure 3: Subject doing self stretching of hamstring muscl

Same procedure was used to measure post intervention assessment for isometric

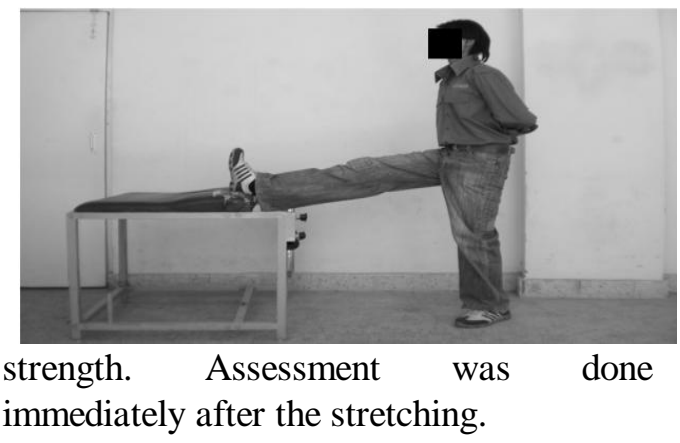

Intervention 
(A). For Hamstring stretching: Subjects were asked to stand facing the wall or quadriceps table, depending on their height. They were asked to do anterior pelvic tilt and then place the heel of the leg to be stretched on the table, and further instructed to keep their hands on their hips, hold their head in a neutral position, look forward, keep the stretched leg fully extended, extend their cervical and thoracic spine and retract their scapulae while maintaining an anterior pelvic tilt. Then they were asked to move their trunks forward at the pelvis until they perceived a hamstring stretching sensation without pain (Fig 3).

(B). For calf stretching: Subjects were made to stand in walk standing position with right lower limb behind the left. Knee of the right lower limb was kept extended; subjects leaned forward over a couch, with foot completely in contact with the ground. They were asked to lean forward till they felt the stretch in calf region but not pain (Fig 4).

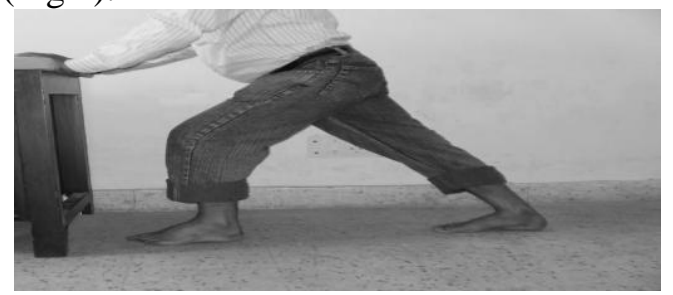

Figure 4: Subject doing self stretching of calf muscle (Runners' stretch)

Protocol of stretching: The subject was asked to stretch the muscle till subject felt the stretch but not pain. This position was instructed to be maintained for 30 seconds and then release it for 20 seconds for a total of 2 minute of time under stretch (Safran, 1989).

Data Analysis
Statistical analysis was done using SPSS 15.0 Software (SPSS Inc., Chicago, USA). "Paired t-test" was used to find difference in strength before and after stretching in each group. For between group analyses an independent t-test was used. A statically significant difference was defined as $\mathrm{p}<0.05$.

\section{Results \& Discussion}

There was a significant difference $(\mathrm{p}<$ 0.05 ) in the means of Pre stretching MIVCF values between Group A and Group B. On the other hand, there was no significant difference $(p>0.05)$ observed in the changes in MIVCF after 2 minute of stretching in Group A and Group B. However, 2 minute stretching of Hamstrings demonstrated an increase (insignificant) in MIVCF by $1.31 \%$, and in calf muscle and MIVCF was found to increase (insignificant) by $2.92 \%$. Between the groups analysis revealed insignificant difference $(p>0.05)$ in the changes in MIVCF after 2 minute of stretching in between Group A and Group B (Table 1).

Table 1: Comparison of mean changes of Maximum Isometric Voluntary Contraction Force (MIVCF) after 2 minute of stretching between group A and group $B$

\begin{tabular}{|c|c|c|c|c|}
\hline & \multirow{2}{*}{$\begin{array}{c}\text { GROUP } \\
\text { A }\end{array}$} & \multirow{2}{*}{$\begin{array}{c}\text { GROUP } \\
\text { B }\end{array}$} & \multicolumn{2}{|c|}{ t-test } \\
\hline & & & $\mathbf{t}$ & $\mathbf{p}$ \\
\hline $\begin{array}{c}\text { Mean changes } \\
\text { in MIVCF } \\
(\mathrm{Kg} .)\end{array}$ & 0.15 & 0.95 & $\begin{array}{c}0.2 \\
8\end{array}$ & $\begin{array}{c}0.47 \\
8\end{array}$ \\
\hline
\end{tabular}

This study was designed to investigate the effect of stretching on reduction of muscle strength and the comparison of a proximal muscle and distal muscles regarding strength loss after stretching. The hypothesis stated that the self-stretching would result in transient 
acute reduction in muscle strength and stretch induced strength loss would be greater for proximal muscle (Hamstring muscle) as compared to distal muscle (Calf muscles). Results of the present study found that shorter stretching (2minute) of hamstring muscle does not have any statistically significant effect on MIVCF although it was found to increase clinically. Therefore, if an athlete stretches his hamstrings prior to sporting event for short duration (2-minute), it will not deteriorate his performance rather it could be beneficial. Shorter stretching (2-minute) of calf muscle resulted in statistically significant increase in MIVCF (11\%); therefore shorter stretching (2-minute) of calf muscle could be beneficial prior to sporting event. By comparing the two results it is concluded that calf muscle seems to be relatively more responsive to stretching than the hamstrings.

In present study stretching of hamstring muscle resulted in increase in MIVCF, which is contrary to most of the previous studies, which have shown acute reductions in muscle strength, but their stretching durations were different from our study (Worrell et al., 1994; Fowles et al., 2000; Herda et al., 2009;). Studies by Fowles et al. (2000), Herda et al. (2009) and Worell et al. (1994) demonstrated significant decreases in strength after 30, 20, 10 minutes of stretching, respectively. Thirty minutes stretching protocol of Fowles et al. (2000) caused greatest decrease in strength $(28 \%$ decrease), followed by 20-min protocol of Herda et al. (2000) that resulted in $10 \%$ decrease and a $10 \mathrm{~min}$ protocol of Worell et al. (1994) resulted in 7\% decrease. However, Reyan et al. (2008) found no changes in voluntary activation (VA) in the plantar flexors when examining the acute effects of only 10-min of stretching, but longer durations of stretching have elicited decreases in VA in the plantar flexors and leg extensors. The findings of the present study are consistent with the findings of Reyan et al (2008) and suggest that shorter durations of stretching do not diminish muscle activation in plantar flexors. No study has compared the acute effects of stretching on proximal and distal muscles. Proximal/larger muscles are sub optimally activated whereas the distal/smaller groups of muscles are fully activated (Kent-Braun and Le Blanc, 1996). Motor unit number is different for proximal and distal muscles. This may be the reason of geting different response from proximal and distal muscles.

Future scope of research

1. EMG could be used in the same study to evaluate action potentials so as to find out the reasons for increase in the strength with 2-min stretching.

2. Since response of stretching may vary with different joint angles and muscle lengths, therefore further studies are needed to compare proximal and distal muscle at different joint angles.

3. More studies are needed to examine athletes under controlled conditions and with sophisticated equipment like dynamometer.

4. Further studies can be done in which the subjects can be recruited with precise amount of muscle tightness, same body stature, and body weight etc.

Conclusion: Shorter stretching (2 minute) increases maximum isometric voluntary contraction force in both muscles but more 
in the calf. This stretching has beneficial effects on muscle performance.

Acknowledgement: Authors acknowledge that there is no conflict of interest of any authors and no funding was provided by any agency for this study.

\section{References}

Behm,, D.G., Button, D.C. and Butt, J.C. 2001. Factors affecting force loss with prolonged stretching. Can. J. Appl. Physiol., 26(3): 261-72.

Bixler, B. and Jonese, R.L. 1992. High-school football injuries: effects of a post-half-time warm-up and stretching routine. Fam. Pract. Res. J, 12: 131-139.

Fowles, J.R., Sale, D.G. and MacDougall, J.D. 2000. Reduced strength after passive stretch of the human plantar flexors. J. Appl. Physiol, 89(3): 1179-88.

Herda, T.J., Ryan, E.D., Smith, A.E, Walter, A.A., Bemben, M.G., Stout, J.R., et al. 2009. Acute effects of passive stretching versus vibration on the neuromuscular function of the plantar flexors. Scand. J. Med. Sci. Sports, 19(5): 703-13.

Kennedy, W.R. 1965. The development and comparison of an electrical strain gauge dynamometer and a cable tensiometer for objective muscle testing. Arch. Phys. Med. Rehabil., 12: 793-803.

Kent-Braun, J.A. and Le Blanc, R. 1996. Quantification of central activation failure during maximal voluntary contractions in humans. Muscle Nerve, 19(7): 861-9.

Knudson, D, Bennett, K., Corn, R., Leick, D. and Smith, C. 2001. Acute effects of stretching are not evident in the kinematics of the vertical jump. J. Strength Cond. Res., 15: 98101.

McHugh, M.P. and Nesse, M. 2007. Effect of stretching on strength loss and pain after eccentric exercise. Medicine \& Science in Sports \& Exercise, 40(3): 566-573.

Ryan, E.D., Beck, T.W., Herda, T.J., Hull, H.R., Hartman, M.J., Stout, J.R. and Cramer, J.T. 2008. Do practical durations of stretching alter muscle strength? A dose-response study. Medicine \& Science in Sports \& Exercise, 40(8): 1529-1537.
Safran, M.R., Seaber, A.V. and Garrett, W.E. Jr. 1989. Warm-up and muscular injury prevention: an update. Sports Med, 8: 239249.

Shellock, F.G. and Prentice, W.E. 1985. Warmingup and stretching for improved physical performance and prevention of sports-related injuries. Sports Med., 2: 267-278.

Shrier, I. 2004. Does stretching improve performance? A systematic and critical review of literature. Clin, J, Sport Med, 14: 267-273.

Smith, C.A. 1994. The warm-up procedure: to stretch or not to stretch. A brief review. J. Orthop. Sports Phys. Ther, 19: 12-17.

Sullivan, M., Dejulia, J.J. and Worrell, T.W. 1992. Effect of pelvic position and stretching method on hamstring muscle flexibility. Med. Sci. Sports Exerc, 24: 1383-1389.

Thacker, S.B., Gilchrist, J., Stroup, D.F. and Kimsey, C.D. Jr. 2004. The impact of stretching on sports injury risk: a systematic review of the literature. Med. Sci. Sports Exerc, 6(3): 371-378.

Tiainen, K., Sipila, S.., Alen, M., and Heikkinen, E. 2004. Heritability of maximal isometric muscle strength in older female twins. $J$. Appl. Physiol., 96: 173-180.

Vivodtzev, I., Pépin, J.L., Vottero, G., Mayer, V., Porsin, B., Lévy, P., 2006. Improvement in Quadriceps strength and dyspnea in daily task after 1 month of electrical stimulation in severely deconditioned and malnourished COPD. Chest, 129(6): 1540-8.

Worrell, T.W., Smith, T.L., and Winegardner, J. 1994. Effect of Hamstring Stretching on Hamstring Muscle performance. J. Orthop. Sports Phys. Therp., 20(3): 154-9.

Worrell, T.W., Sullivan, M.K., and Dejulia, J.J. 1992. Reliabilty of an active knee extension test for determining hamstring muscle flexibility. J. Sport Rehabil., 1:181-187.

Young, W. and Elliott, S. 2001. Acute effects of static stretching, proprioceptive neuromuscular fascilitation stretching, and maximum voluntary contractions on explosive force production and jumping performance. Res. Q. Exerc. Sport, 72: 273-279. 